\title{
Il poeta in pubblico negli anni Settanta
}

\author{
Giovanna A. Wedel
}

\begin{abstract}
Dagli albori del secolo si discute se la poesia sia dentro o fuori.

Dapprima vinse il dentro, poi contrattaccò duramente il fuori e dopo anni si addivenne a un forfait che non potrà durare perché il fuori è armato fino ai denti. (604)
\end{abstract}

Con questi versi tratti dal Quaderno di quattro anni del 1977, Montale riflette sul fenomeno di svalutazione del codice di scrittura poetica verificatosi negli anni Settanta a favore di una semantizzazione possibile oltre i limiti del testo. Tale osservazione metatestuale si inserisce nel lungo dibattito sullo sviluppo e sul rinnovamento della poesia, apertosi nel dopoguerra e non ancora concluso. Le radici dell'attuale e a lungo discussa crisi della poesia sono da ricercarsi già nelle propaggini dell'Ermetismo estentuato e scaduto ad eloquenza di maniera; ad esso si opposero i poeti della quarta generazione che produssero una poesia dal tono minore, operando una demistificazione estetica della lingua e aprendo la via agli sperimentalismi. Le esperienze sempre più radicali dell'avanguardia degli anni Sessanta che determinarono un allontanamento deciso dei lettori, suscitarono in tutti il sospetto che la poesia, ormai priva di un messaggio significante, avesse perduto ogni funzione o ragione d'essere in una società tecnologica e di comunicazioni di massa. E alla fine degli anni Sessanta l'estrema riduzione sensica e segnica subita dal linguaggio diffuse una sfiducia generalizzata nelle possibilità comunicative e nella validità della scrittura poetica.

Successivamente, nel tentativo di legittimare la propria sopravvivenza e di instaurare un rapporto più diretto col pubblico, il poeta ha adottato mezzi linguistici più aderenti alla nuova realtà industriale, ha allargato gli spazi tradizionali della pratica poetica e ha ottenuto dei risultati artistici che richiedono da parte del destinatario un atto di fruizione più attivo e pluridimensionale. In proposito si possono menzionare gli esperimenti di scrittura simbiotica di Ugo Carrega e Arrigo Lora-Totino, che consiste nell'interazione di elementi fonetici, grafici e visivi; la poesia totale di luciano Caruso e Adriano Spatola, fondata sull'esercizio gestuale, invece che letterario e mirante alla fusione di tutte le arti; o ancora la poesia ginnica di Lora-Totino, risultante dall'integrarsi delle parole con la mimica. Ma è soprattutto in numerose manifestazioni 
teatralizzate degli anni Settanta che la poesia oltrepassa $i$ confini istituzionalizzati della pagina. In letture poetiche alla radio, alle stazioni televisive, in sale private e in piazza, il testo passa in secondo piano o scompare del tutto di fronte alla testimonianza orale, al mezzo musicale o al gesto fisico del poeta.

Tale fenomeno che si è diffuso in numerosi paesi del mondo (tra cui la Russia, la Germania, la Francia ecc.) è stato avviato in Italia dalle celebri serate svoltesi dal 5 febbraio al 28 maggio 1977 al "Beat '72," un teatro off-off di Roma. Seguirono fra manifestazioni più memorabili, le letture al Salone Pier Lombardo di Milano nel maggio 1977, il Festival di Poesia Internazionale di Rotterdam, in giugno, a cui partecipò anche l'Italia; la Seconda Rassegna Internazionale della Performance dal $1^{\circ}$ al 6 giugno 1978, organizzata da Barilli a Bologna; le rappresentazioni teatrali della Sex Poetry, animate da Michelangelo Coviello e altri a Milano, tra i mesi di gennaio e marzo 1979; la Settimana Genovese della Poesia in Pubblico, organizzata da Sanguineti tra il 21 e il 27 maggio 1979 nel quartiere di Sampierdarena, e infine il Festival Internazionale dei Poeti, organizzato dall'assessore Nicolini sulla spiaggia libera di Castelporziano a Ostia, tra il 28 e il 30 giugno 1979. Numerosi sono poi i salotti di lettura e le premiazioni pubbliche che tutt'ora si svolgono in centri grandi e piccoli d'Italia. In queste manifestazioni la poesia si trasforma in un evento multimediale allestito per una ricezione collettiva simultanea. Il poeta si presenta in veste istrionica esibendosi come interprete piuttosto che come autore dei propri versi e proponendo un ruolo sociale facilmente scadente nel divismo più mondano. Egli cerca di strappare il consenso del pubblico adoperando la parola poetica come uno degli elementi strutturali dello spettacolo e presentandosi come soggetto ed oggetto dell'enunciazione nel rapporto interlocutivo col destinatario. L'attenzione degli spettatori viene allontanata dal segno grafico e deviata dal referente ad un falso emittente; il messaggio originario viene soppiantato da un secondo messaggio la cui codificazione dipende in gran parte dal pubblico ricevente. Gli elementi del processo di comunicazione divengono quindi ambigui e mutevoli e l'esito semantico dello spettacolo rimane completamente al di fuori dell'area linguistica.

Nella cantina del "Beat '72" ogni sabato sera un poeta diverso entrava in scena per proporre ad un pubblico brechtianamente partecipe uno spettacolo in gran parte improvvisato. La comparsa del poeta, attore e interprete del proprio personaggio, non era nuova. Frequenti letture di versi si erano già verificate alla fine del Settecento; nel secolo successivo Edgar Allan Poe aveva attraversato l'America settentrionale leggendo "Il corvo"; nel nostro secolo si possono rammentare le serate dadaiste al Cabaret Voltaire, certi avvenimenti scenici futuristi, in cui confluivano strutture artistiche diverse per realizzare una forma teatrale dinamica, sintetica e che coinvolgesse gli spettatori; e ancora le serate della Beat Generation americana (con le letture di Ginsberg a San Francisco) e le letture in pubblico di Evtuscenko. In tutti i casi è 
presente l'intento demistificante della figura del poeta e popolarizzante della poesia, che, tradizionalmente, era sempre stata ritenuta una pratica privata e spesso elitaria. I luoghi del teatro vengono adoperati con fini propagandistici per comunicare l'esistenza del poeta o di un movimento poetico, ma non per trasmettere un messaggio lirico preciso. L'insuccesso dimostrato dall'editoria nella diffusione della poesia a livello di massa ha spinto il poeta ad un confronto diretto col pubblico, che, però, in assenza di coefficienti mediatori, provoca una ricezione troppo immediata e impersonale; il pubblico incapace di assimilare o riconoscere il messaggio poetico, dinanzi ad uno spettacolo che non corrisponde alla sua aspettazione, reagisce con un atteggiamento di disinteresse o di rifiuto.

Gli esperimenti di poesia teatralizzata degli anni Settanta attuano numerose proposte formulate nci manifesti teatrali futuristi, dal "Teatro di Varietà" al "Teatro della Sorpresa." Marinetti sosteneva, ad esempio, di voler distruggere "il Solenne, il Sacro, il Serio, il Sublime dell'Arte coll'A maiuscolo" (115), di utilizzare la collaborazione rumorosa e spesso ostile del pubblico e di servirsi dell'improvvisazione per divertirlo con "effetti di comicità, di eccitazione erotica o di stupore immaginativo" (112). Come già nelle serate futuriste, la linea di demarcazione fra Arte e Vita si annulla nella realtà corporea del poeta. La suddivisione spaziale fra palcoscenico e platea scompare in un happening collettivo che si svolge all'intero dell'interno teatro e al di fuori dello spazio poetico testuale. Però, mentre negli spettacoli futuristi si mirava a sfidare la reazione del pubblico attraverso la recitazione del testo poetico, nelle letture degli anni Settanta il messaggio lirico verbale scompare nell'interazione con aree semantiche diverse. Ciò si può riscontrare nel seguente resoconto giornalistico della serata d'apertura al "Beat '72," apparso su Il Messaggero:

Alle 22,30 un folto pubblico amante della pocsia aspettava Dario Bellezza (il poeta di turno) con l'annunciato spettacolo ispirato alle sue poesie "Sodoma ad Auschwitz." Alle 22,30 gli organizzatori degli incontri, Franco Cordelli, Simone Carella e Ulisse Benedetti si vedevano costretti a comunicare l'assenza di Bellezza per motivi imprecisati. Il pubblico reagiva con sdegno da una parte (un gruppo di poeti napoletani crano venuti da Napoli solo per assistere alla scrata) e con reverenza dall'altro (ci si interrogava sul significato profondo del gesto provocatorio del Poeta).

\section{$(\ldots)$}

A Mezzanottc c dieci primo colpo di scena: arriva Bellezza e il pubblico che cominiciava ad andarsene torna di corsa a prendere posto. Rifiuta di dare spiegazioni del ritardo, ma si sicde al tavolino verde con fiorellini bianchi preparato per l'occasione e dà lettura di alcune poesie scelte dalle raccolte "La morte segreta" e "Invettive e licenze."

A Mezzanotte e trenta secondo colpo di scena: fa irruzione Riccardo Corso, attore c regista del mancato spettacolo "Sodoma ad Auschwitz," simultaneamente sferra un pugno al tavolo che precipita verso il pubblico e un calcio al Poeta che rotola con sedia e poesia sul pavimento. Corso afferra il microfono e rivela i retroscena degli sconcertanti avvenimenti agli sconcertati spettatori. 


\section{(...)}

Grida c richicste di ulteriori spicgazioni da parte del pubblico. I Napoletani insistono nel voler ascoltare Ie poesic di Bellezza. (...) Il Pocta riprende a leggere massaggiandosi il fianco colpito. Riccardo Corso si drappeggia addosso veli azzurri c dà vita con un partner a una scena dell'incriminato spcttacolo: masturbazioni c dolci abbandoni fra omosessuali. Voci dal pubblico: "Ma che fanno quei due? Perché nun se nc vanno?" All'una c un quarto lo spellacolo è finito. Bellczat commenta: "Non ho proprio niente da spicgare. Mi c̀ stata fatta violenza. So solo questo. (Pctrignani)

È possibile osservare come l'intenzione autodissacrante del poeta venga pienamente realizzata attraverso uno spettacolo in larga parte gestuale e di sorpresa, di cui il testo scritto costituisce un fattore facilmente trascurabile. Nel tentativo di socializzare il proprio ruolo e di dialogare a tutti i costi col lettore disattento, il poeta finisce col negare la "poesia" intesa come forma d'arte fissa ed eternamente fruibile, dando vita ad un evento effimero ed irripetibile, e perciò fondamentalmente anti-artistico, di cui il pubblico è co-autore. Ogni elemento d'improvvisazione per quanto patetico o scandaloso diviene una componente della rappresentazione che assume risvolti simbolici imprevisti. Ed ecco che il ritardo del poeta punta alla sua diffidenza nella capacità di comunicare col pubblico, o anche solo di scoprire la sfera del privato; i commenti ironici degli spettatori manifestano l'incomprensione e il rifiuto di una situazione poetica equivoca, dove il testo si disintegra in un procedimento teatrale in continua (tras)formazione. E lo smitizzante calcio ricevuto da Bellezza sul palcoscenico sottolinea il ruolo vittimistico e autodegradante assunto dal poeta, e allo stesso tempo costituisce la risposta al suo gesto provocatorio. Altrettanto emblematici ed eversivi furono in successive serate al "Beat '72" la comparsa in scena di Paolo Prestigiacomo per mezzo di diapositive rappresentanti la propria immagine, e con la propria voce registrata su nastri; il gesto incendiario di Valentino Zeichen e Franca Rovigati, i quali bruciarono simbolicamente la poesia in forma di barchette di carta; e l'atto sfrontato di Cesare Viviani, il "Pescicida" (nella definizione di Cordelli) che decapitò pesciolini rossi gettandoli poi in faccia agli spettatori. Non mancò il poeta che rimase in silenzio per mezz'ora di fronte al pubblico, mimando cosi la situazione poetica dell'ultimo ventennio. Mariella Bettarini, nel tentativo di liberare poeta, pubblico e poesia dal contesto tradizionale della sala da lettura, caricò gli spettatori su due autobus e li potrò a Fiumicino, leggendo poesie al microfono. Si trattò, cioè, di spettacoli che si mantennero in bilico fra il gioco e la beffa, il riso e l'ironia, e il cui aspetto aggressivo riporta all'operazione simbolica di rivolta degli spettacoli dadaisti. Anche il Dadaismo aveva tentato di ristabilire il contatto col pubblico andato perduto verso la metà dell'Ottocento, e aveva sostituito alla classica staticità auratica dell'arte il gesto scandaloso e provocatore a cui il destinatario doveva reagire polemicamente. Se, però, gli spettacoli d'avanguardia della prima metà del secolo vennero seguiti da un pubblico vasto, le manifestazioni di poesia 
teatralizzata degli anni Settanta non riuscirono a suscitare grande interesse o entusiasmo in Italia. Le serate al "Beat " 72 " dopo le settimane iniziali furono poco frequentate; le serate al Salone Pier Lombardo di Milano e alla Seconda Rassegna Internazionale della Performance (dove per uscire dalla pagina si fecero vari esperimenti di poesia sonora e gestuale) non si poterono assolutamente chiamare eventi di massa; in una lettura di Adriano Spatola nel 1979, ad Abano Terme, pare che l'unico spettatore fosse costituito dal gestore del locale. A Castelporziano, dove accorsero invece migliaia di persone da tutto il mondo, l'accoglienza ai poeti fu tutt'altro che lusinghiera. La prima giornata si concluse con un pandemonio di persone che gridavano, fischiavano, lanciavano sabbia e bucce di cocomero, privando i poeti del privilegio della parola. Il pubblico reagiva, cioè, con un atteggiamento di energico rifiuto e contestazione a priori. A differenza dei futuristi che erano riusciti a declamare i propri versi e a trasformare la violenza delle proprie serate in un ingrediente di successo, i poeti di Castelporziano furono inequivocabilmente messi a tacere e spogliati della funzione sociale. La frattura comunicativa col pubblico fu completa, mentre all'attenzione per la parola poetica sottentrò lo scherno dell'esaltazione esibizionistica del corpo del commediante.

Autogestendosi il poeta ha scoperto la paradossale inutilità e insignificanza del proprio ruolo di scrittore. In scena la poesia non consiste né nel rapporto semantico e fonetico dei segni sulla pagina, né nel rapporto audio-visivo stabilito fra il lettore e il suo pubblico, bensì nella parte di un copione o addirittura di un canovaccio da utilizzare in una rappresentazione drammatica mancata. L'unità del messaggio poetico si frantuma in infinite possibilità espressive dei poeti-attori, e ricettive del pubblico, per l'ovvia irrepetibilità della rappresentazione. Anche in una società di comunicazioni di massa, per risvegliare l'interesse del lettore, il poeta deve restare con discrezione dietro le quinte di strutture poetiche costruite per il rituale apprezzamento del destinatario sconosciuto. L'operazione poetica, pur nel suo costante moto di rinnovamento e di ricerca sperimentale, deve rimanere fondamentalmente una pratica linguistica, che può arricchirsi di novità tecniche interdisciplinari, senza scomparire nei canali della comunicazione di massa; il pericolo da evitare è quello di scambiare gli strumenti poetici per il prodotto.

\section{Indiana University}

\section{OPERE CITATE}

Marinetti e il Futuriamo. A cura di Luciano De Maria. Milano: Mondadori, 1973.

Montale, Eugenio. "La poesia." Tutte le poesie. A cura di Giorgio Zampa. Milano: Mondadori, 1984. 604.

Petrignani, Sandra. "Preso a calci il poeta dal regista infuriato." I/ Messaggero 8 febb. 1977. Cit. in Anfonio Barburo. Da Narciso a Castelporziano. Roma: Edizioni dell'Ateneo, 1981. 117-8. 